\title{
Correspondence
}

\section{The writings of Thomas Szasz}

Dr Benning ${ }^{1}$ nicely summarises some of the major conceptual errors in the writings of the late Dr Thomas Szasz.

Dr Szasz, who was one of my professors during residency, had important things to say about protecting the civil liberties of people with mental illness. However, his view of schizophrenia as a self-inflicted form of lying has done great injury to those who have this devastating illness. For example, in his 1996 book The Meaning of Mind, Szasz wrote:

'I believe viewing the schizophrenic as a liar would advance our understanding of schizophrenia. What does he lie about? Principally about his own anxieties, bewilderments, confusions, deficiencies and self-deception'2 (p. 130).

In recent years Szasz's position has been undermined by scores of studies showing that individuals diagnosed with schizophrenia show brain abnormalities at a significantly higher frequency than healthy controls. ${ }^{3-5}$ More important, however, is the recognition that disease (dis-ease) is best understood as an enduring state of suffering and incapacity not, as Szasz argued, as the presence of lesions or abnormal physiology. ${ }^{6}$

Ronald W. Pies, Professor of Psychiatry, SUNY Upstate Medical University, Syracuse, New York and Tufts University School of Medicine, Boston, USA; email: ronpies@massmed.org

1 Benning TB. No such thing as mental illness? Critical reflections on the major ideas and legacy of Thomas Szasz. BJPsych Bull 2016; 40: 292-95.

2 Szasz TS. The Meaning of Mind: Language, Morality and Neuroscience. Praeger, 1996.

3 Bakhshi K, Chance SA. The neuropathology of schizophrenia: a selective review of past studies and emerging themes in brain structure and cytoarchitecture. Neuroscience 2015; 303: 82-102.

4 Woo TU. Neurobiology of schizophrenia onset. Curr Top Behav Neurosci 2014; 16: 267-95.

5 Iritani S. What happens in the brain of schizophrenia patients? An investigation from the viewpoint of neuropathology. Nagoya J Med Sci 2013; 75: 11-28.

6 Pies R. On myths and countermyths: more on Szaszian fallacies. Arch Gen Psychiatry. 1979; 36: 139-44.

doi: $10.1192 / p b .41 .2 .120$

\section{Critical reflections on psychiatry: could Thomas Szasz still have relevance in modern medical practice?}

Tony B. Benning provides a considered review of the main arguments proposed by Thomas Szasz and explores their relevance in the present day. ${ }^{1}$ On the surface, many of Szasz's arguments are easy to refute: Benning cites Kendell, ${ }^{2}$ who draws attention to areas of similarity between medical and psychiatric diagnoses - for example, the importance of distress and impairment of function in the definition of illness. Szasz's criticism of the nature of boundaries between mental health and illness can also be applied to physical health conditions such as type 2 diabetes and hypertension, in which pathology is determined to occur at the extremes of continuous variables.
As with psychopathology, the cut-off point at which it is believed there is a risk to well-being significant enough to justify intervention is to some extent arbitrary, as evidenced by intermittent changes in international definitions of the thresholds for diagnosing and treating these conditions.

However, as Benning points out, psychiatrists might do well to bear in mind some of Szasz's concerns, particularly those around the interlinking themes of personal responsibility and psychiatric power. It may be clear to those who have witnessed the deviation from the authentic self (encountered in severe mental illness) that sufferers lack capacity for meaningful autonomous decision-making and that a duty exists for doctors to treat in their best interests as they would for any critical illness. At the same time, it is widely acknowledged that a diagnosis of mental illness does not necessarily entail incompetence, as acknowledged by the Mental Health Act's consideration of patients deemed to have the capacity to refuse treatment while detained. ${ }^{3}$ I would argue that where clinicians believe an individual retains the capacity for autonomy, exercising their legal power to detain and compulsorily treat against the individual's wishes goes against the fundamental tenets of medical ethics and violates the doctor-patient relationship. Szasz's dismissal of psychiatrists as agents of a therapeutic state appears to have some traction here.

Another area of continuing relevance is Szasz's condemnation of the pathologisation of human experience. It is, arguably, beneficence that drives the profession to try to alleviate - through diagnosis and therapeutic intervention suffering that in part originates from the experience of social adversity. Delgadillo et al highlight the increased prevalence of mental ill health in more economically deprived areas and the lower rates of recovery found in these populations; ${ }^{4}$ clinical commissioning groups responsible for the local provision of psychological therapies are categorised as underperforming with regards to the latter.

However, when health services are blamed for patients failing to improve, society effectively abdicates from its responsibility to address the inequalities and social ills that may explain distress better than any medical nosology, as Szasz contended. Under these circumstances the psychiatric profession may not be pursuing its own political agenda so much as being caught up in a greater one, which might potentially overwhelm its capacity to function in the current climate of financial constraint. Ironically, Szasz's criticism of the pathologising of day-to-day life also serves to draw further parallels between physical and mental illness. Take, for example, obesity, which the medical profession are being increasingly held responsible for addressing, despite there being clear social determinants. ${ }^{5}$ An awareness of professional boundaries and limitations could avoid compromising the delivery of care in areas that are clearly within the medical sphere, and challenge any attempt to avoid responsibility for societal well-being by other sectors such as welfare and housing.

Thus, while Benning sets out many arguments that demonstrate how Szasz might be wrong, he is pertinent in 